\title{
Asymmetry in transitional pipe flow of drag-reducing polymer solutions
}

\author{
M.P. Escudier*, S. Rosa, R.J. Poole \\ University of Liverpool, Department of Engineering, Liverpool L69 3GH, UK
}

\begin{abstract}
A B S T R A C T
New experimental data are presented and discussed for fully developed pipe flow of shear-thinning, viscoelastic polymer solutions in the transitional regime between laminar and turbulent flow. The data confirm that such transitional flows exhibit significant departures from axisymmetry in contrast to the fully developed pipe flow of Newtonian fluids or both laminar and turbulent flows of such drag-reducing liquids. The azimuthal structure of the asymmetry is investigated together with its axial development and also the velocity fluctuation levels. These data do not lead to an explanation for the asymmetry but do suggest that the influence of the flow geometry both upstream and downstream can be ruled out.
\end{abstract}

\section{Introduction}

Significant departures from axisymmetry in the mean velocity profiles for "fully developed" pipe flow of non-Newtonian liquids in the transitional region between laminar and turbulent flow conditions were first reported [1] for the flow of Laponite, a shear-thinning, thixotropic synthetic clay with a yield stress. Subsequently [2,3] it became apparent that such velocityprofile asymmetry was evident for a wide range of drag-reducing (shear-thinning and viscoelastic) polymer solutions: xanthan gum, polyacrylamide, and CMC, and also for Carbopol [3], a shearthinning, yield-stress fluid. Unless intentionally provoked, for example by an imposed asymmetric disturbance upstream [4], the time-averaged velocity profiles for fully developed transitional pipe flow of Newtonian fluids invariably are found to be axisymmetric within experimental uncertainty (see e.g. $[2,5,6]$ ). The fact that similar behaviour was observed for non-Newtonian liquid flows in three completely independent research laboratories, in France, Australia and UK, led to the conclusion [2] that a fluid-dynamic mechanism was more likely to be responsible for the asymmetry than imperfections in the flow facilities. Further observations of asymmetry in the transitional pipe flow of shear-thinning polymers have been reported recently by a group in Canada [7,8] and again the conclusion reached was that the asymmetry is a fluid-mechanical phenomenon.

For fully developed laminar pipe flow, asymmetry can arise as a consequence of secondary flows driven by the Coriolis acceleration due to the Earth's rotation if the Ekman number (the ratio of viscous to Coriolis forces) is sufficiently low [9]. It is important to note the restriction to fully developed laminar flow where there is a simple balance between viscous forces and pressure gradient while inertia plays no role. In transitional and turbulent flows, inertia is far more important, even though the mean flow may be fully developed, and the flow is unaffected by the Coriolis acceleration even where the Ekman number is low. Under these circumstances, the Rossby number (the ratio of inertia to Coriolis forces) becomes the governing parameter [9]. Secondary flows, caused by curvature of the pipe axis, can also distort the mean velocity profiles in laminar flow if the curvature is sufficient to lead to high values of the Dean number [10]. The time-averaged velocity profiles for fully developed turbulent pipe flow, Newtonian and non-Newtonian alike, are found invariably to be axisymmetric (see e.g. [6] or [11]).

It is immediately obvious that a velocity profile which is asymmetric in a single measured plane must exhibit an azimuthal variation but only recently [12] has this been demonstrated experimentally (for Carbopol). Apart from the latter paper, all previous observations of transitional pipe flow were an incidental aspect of investigations into fully developed turbulent flow of drag-reducing polymer solutions. In the present paper we present detailed data for transitional pipe flow of a polyacrylamide and a xanthan gum. In an attempt to identify the underlying cause of asymmetry, we explore the influence of imposing both upstream and downstream distortion on the flow. We introduce a parameter $\alpha$ to quantify the level of azimuthal asymmetry and show how the asymmetry evolves with streamwise distance along the pipe. This parameter is defined as

$\alpha=\frac{2 \pi \int_{0}^{R}\left(u-u_{M}\right) \cdot r \cdot \mathrm{d} r}{2 \pi \int_{0}^{R} u_{M} \cdot r \cdot \mathrm{d} r} \times 100$ 
where $u_{M}$ is the arithmetic mean of the two values of the mean velocity $u$ at the same radial location $r$ on either "side" of the pipe centreline (i.e. two points $180^{\circ}$ apart). The intention of the $r$ weighting is to introduce a flowrate effect (the denominator of Eq. (1) is simply the volumetric flowrate if the flow is axisymmetric). After inspecting many velocity profiles we conclude that the threshold for considering a profile to be considered significantly asymmetric corresponds with a value of $\alpha$ of $2 \%$ and for $\alpha$ less than $1 \%$ any asymmetry is practically imperceptible. An alternative approach [12] to quantifying the asymmetry is in terms of the streak velocity at a particular radial location defined as the difference between the mean velocity at that radius and the azimuthally averaged mean velocity at the same radius.

\section{Experimental flow facility and instrumentation}

The flow facility used for the measurements reported here is a modified version of that used in our previous research [1] in that the pipe has been extended from about $13 \mathrm{~m}$ to $23.3 \mathrm{~m}$ overall length. Apart from a $1.72 \mathrm{~m}$ section downstream of the plenum chamber, the pipe run consists of 21 modules each $1.027 \mathrm{~m}( \pm 3 \mathrm{~mm})$ long and $100.4 \pm 0.1 \mathrm{~mm}$ internal diameter. Each module is made up of a length of precision-bore borosilicate glass tube with a stainless steel flange at either end. The glass tube is separated from the stainless steel by a thin PTFE ring and each end of the module (assembled in a vertical jig to ensure concentricity between the bore of the glass tube and that of the flange) is fixed using Devcon urethane rubber. To ensure the best-possible matching and so minimise disturbances to the flow, the flanges were machined in male/female pairs. To avoid any possibility of longitudinal-curvature effects leading to secondary flows, the pipe run was assembled guided by a laser beam/target process which ensured overall end-to-end straightness well within $1 \mathrm{~mm}$. In addition to the extended length, a major modification to the flowloop was a plenum chamber introduced specifically to minimise swirl and ensure uniform flow at the pipe inlet. As can be seen in the schematic diagram of the plenum chamber, Fig. 1, flow enters the plenum chamber through a $100 \mathrm{~mm} \varnothing$ pipe which turns through $90^{\circ}$ so that the flow is directed towards the back wall of the chamber. The disc with four $57.7 \mathrm{~mm} \varnothing$ holes at $90^{\circ}$ intervals around its centre and four peripheral cutouts is intended to distribute the flow prior to the inlet contraction and flow straightener. More complete details of the unmodified flow facility are given in [1]. In the discussion below, we refer to the angle $\theta$ defined with respect to the vertical $\left(\theta=0^{\circ}\right)$, as shown in Fig. 1.

Pressure differences were measured between tappings $14 \mathrm{~m}$ and $21 \mathrm{~m}$ from the pipe inlet from which an average wall shear stress was determined for each flow condition. Pressure tappings $1 \mathrm{~mm}$ in diameter are located on each flange pair and connected to a Druck model LPX9381 differential pressure transducer (full scale 50 mbar) using $2 \mathrm{~mm}$ ID clear vinyl tubing filled with the working fluid. The bulk flowrate $\dot{m}$ was measured with an uncertainty less than $1 \%$ using an Endress + Hauser Coriolis mass flowmeter, model Promass 63.

Mean velocity and axial-velocity fluctuations ("turbulence intensities" or Reynolds normal stresses) were estimated using a Dantec Fibreflow laser Doppler anemometer (LDA) system operated in forward scatter. The transmitting optics comprised a $60 \times 10$ probe incorporating a 55X12 beam expander with a S055X0342 receiving optics. The beam separation at the front lens was $40 \mathrm{~mm}$ and the lens focal length $160 \mathrm{~mm}$ which produces a measurement volume $20 \mu \mathrm{m}$ in diameter and $0.21 \mathrm{~mm}$ in length. Given such a short measuring volume and the large pipe diameter, no correction was made for gradient broadening. Doppler signals were processed using a model 57N25 burst spectrum analyser. Mean velocities and
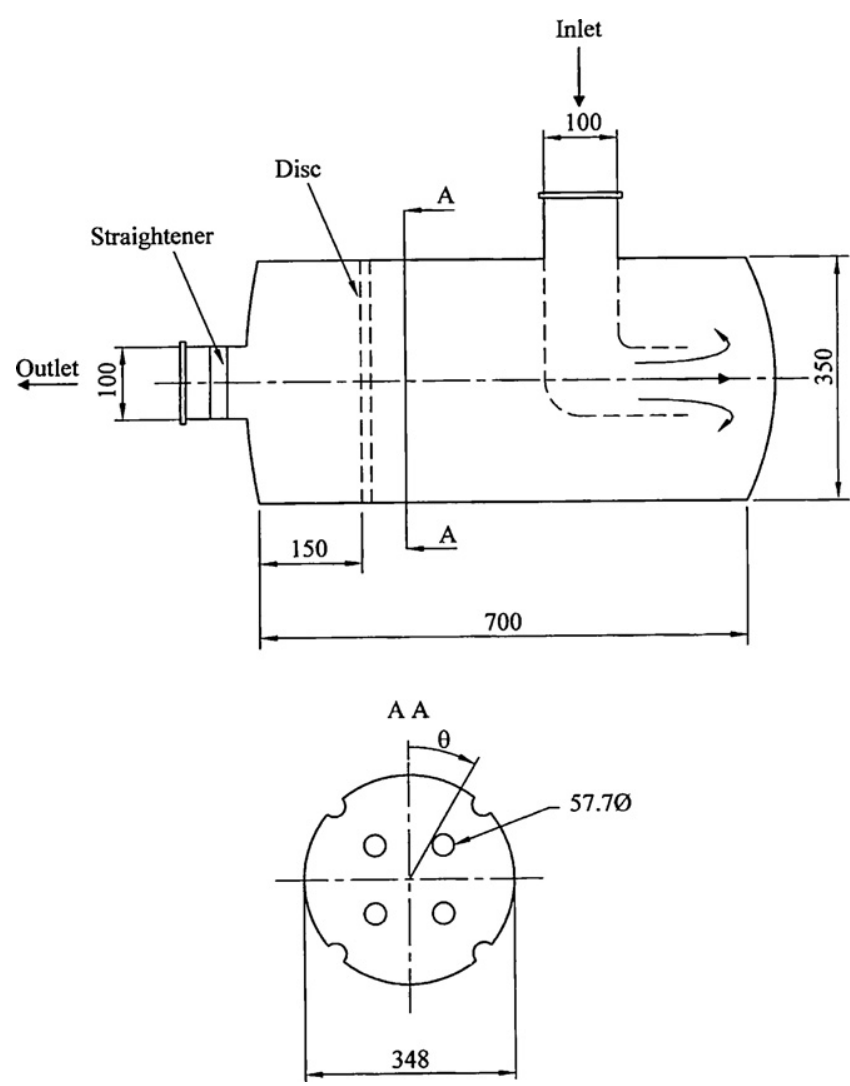

Fig. 1. Plenum chamber schematic with details of the perforated disc and definition of the angle $\theta$ (dimensions in $\mathrm{mm}$ ).

rms fluctuation intensities were determined by averaging data from typically 20,000 samples collected at each radial measuring location so that a velocity profile consisting of 50 points took between $3 \mathrm{~h}$ and $4 \mathrm{~h}$ given a data rate of typically $80 \mathrm{~Hz}$. Velocities were calculated using transit-time weighting [13]. The estimated overall uncertainty is $3 \%$ in the mean velocity and $6 \%$ in the turbulence intensity. The flows were seeded with Timiron particles, ca $5 \mu \mathrm{m}$ in diameter, Supersilk MP-1005 supplied by S Blanck Ltd. A specially constructed traverse system permitted radial traverses of the LDA measuring volume at multiples of $15^{\circ}$ to the vertical (taken as $0^{\circ}$ ).

\section{Fluid rheology}

Preliminary experiments were carried out with a Newtonian fluid, a $60 \%(\mathrm{w} / \mathrm{w})$ aqueous solution of glycerol which had a measured viscosity at $20^{\circ} \mathrm{C}$ of $0.00858 \mathrm{~Pa}$ s and a density of $1140 \mathrm{~kg} / \mathrm{m}^{3}$. Two of the non-Newtonian working fluids were $0.03 \%(\mathrm{w} / \mathrm{w})$ $(300 \mathrm{ppm})$ and $0.125 \%(\mathrm{w} / \mathrm{w})$ aqueous solutions of a polyacylamide (hereafter referred to as PAA) with a molecular weight of $1.9 \times 10^{6} \mathrm{~g} / \mathrm{mol}$, Separan AP273E supplied by Floerger. The third fluid was a $0.15 \%$ aqueous solution of a xanthan gum (hereafter XG) with a molecular weight of $5.1 \times 10^{6} \mathrm{~g} / \mathrm{mol}$, Keltrol TF supplied by Nutrasweet-Kelco Ltd. The solvent was tap water with $100 \mathrm{ppm}$ $40 \%(w / w)$ solution of formaldehyde added as a biocide. PAA is shear thinning, regarded as having a highly flexible molecular structure [14] and so is highly viscoelastic and drag reducing. XG is also shear thinning, but has more of a rigid-rod structure and so is less viscoelastic and drag reducing [15]. The two concentrations of PAA were selected as, at these concentrations, both solutions are highly effective drag-reducers-producing drag-reduction levels close to the maximum asymptote [16] regardless of pipe diameter, yet they exhibit significantly different levels of shear thinning. The concen- 


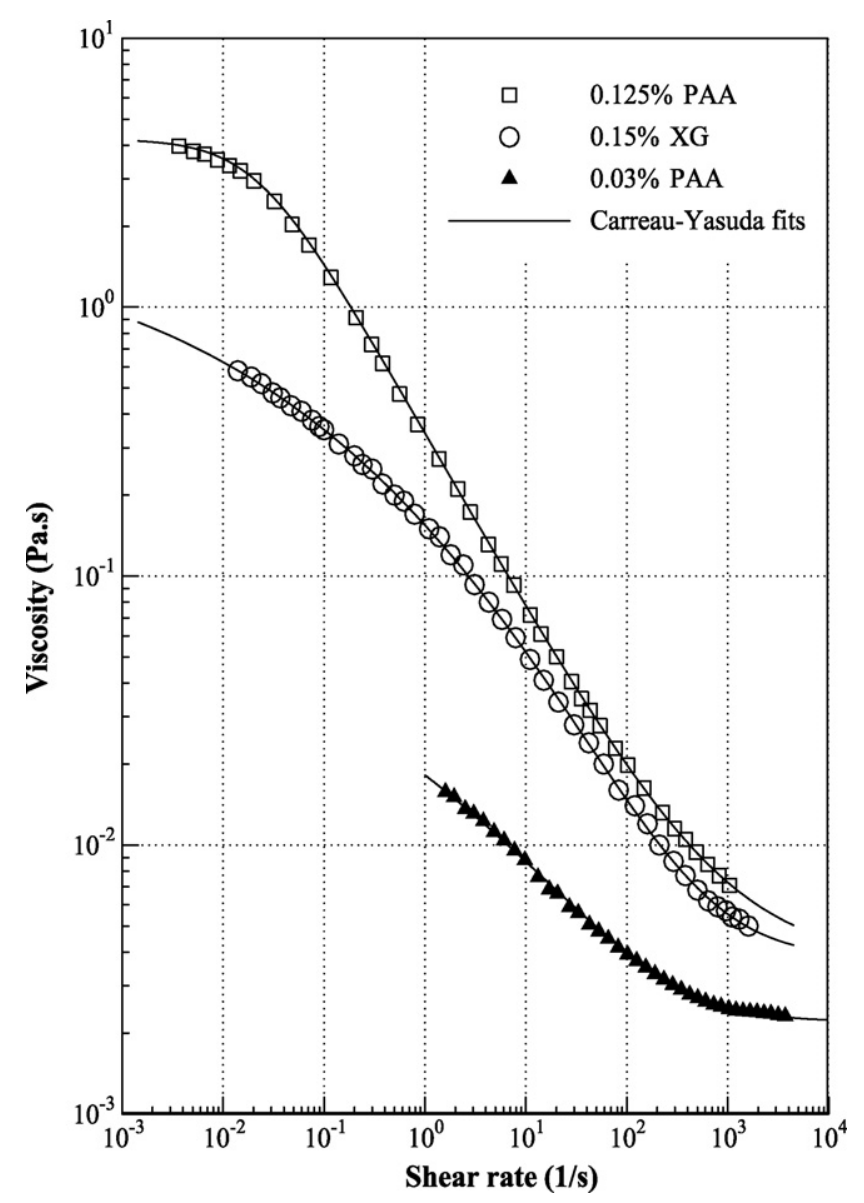

Fig. 2. Flow curves for $0.03 \% \mathrm{PAA}, 0.125 \% \mathrm{PAA}$ and $0.15 \% \mathrm{XG}$. Solid curves represent the Carreau-Yasuda model.

tration levels of the $0.125 \%$ PAA and $0.15 \%$ XG were chosen with the intention of matching the shear-thinning characteristics of two fluids which are known to exhibit significantly different levels of elasticity [14].

The flow curves (shear viscosity vs shear rate) shown in Fig. 2 for the polymer solutions were determined at $20^{\circ} \mathrm{C}$ using a TA Instruments Rheolyst AR1000N controlled-stress rheometer using a double-concentric cylinder geometry. The smooth curves in Fig. 2 represent the Carreau-Yasuda [17] model

$\mu_{C Y}=\mu_{\infty}+\frac{\mu_{0}-\mu_{\infty}}{\left(1+\left(\lambda_{C Y} \dot{\gamma}\right)^{a}\right)^{n / a}}$

which is an excellent fit to each set of data. In Eq. (2), $\mu_{0}$ is the zero-shear-rate viscosity, $\mu_{\infty}$ is the infinite-shear-rate viscosity, $\lambda_{C Y}$ is a constant (with dimensions of time) which can be regarded as characterising the shear rate for the onset of shear thinning, $n$ is a power-law index and $a$ is a fitting parameter introduced by Yasuda et al. [17]. The Carreau-Yasuda parameters are listed in Table 1. A plot of $\mu_{0}$ vs concentration $c$ in $\log -\log$ form [18,19], Fig. 3, shows well-defined power-law variations, $\mu_{0} \propto c^{m}$, with different values of the exponent $m$ for the two polymers above and below the critical overlap concentration $c^{*}$. For solutions with $c<c^{*}$ the

Table 1

Carreau-Yasuda parameters for all liquids investigated.

\begin{tabular}{llllll}
\hline Fluid & $\mu_{0}($ Pas $)$ & $\mu_{\infty}($ Pas $)$ & $\lambda_{C Y}(\mathrm{~s})$ & $n$ & $a$ \\
\hline $0.03 \%$ PAA & 0.115 & 0.00221 & $10^{-6}$ & 3.90 & 0.173 \\
$0.125 \%$ PAA & 4.22 & 0.00372 & 45.8 & 0.660 & 1.25 \\
$0.15 \%$ XG & 1.95 & 0.00382 & 0.0161 & 1.38 & 0.198 \\
\hline
\end{tabular}

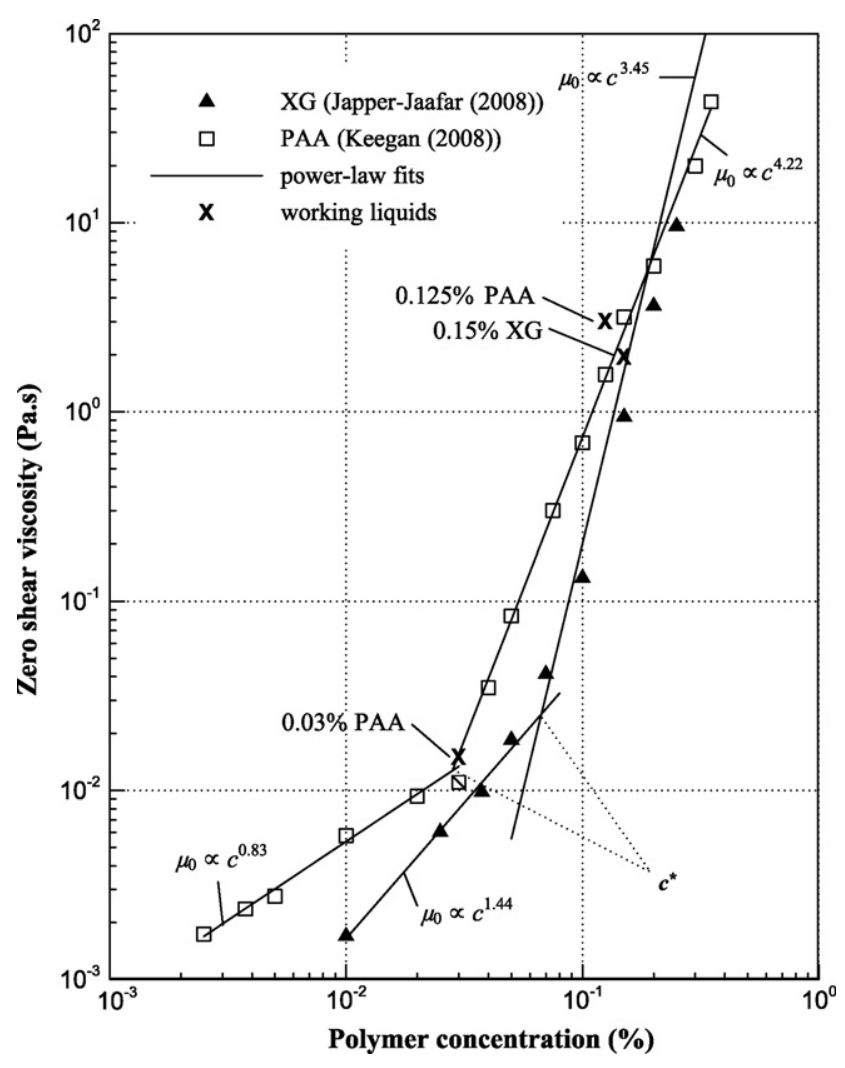

Fig. 3. Zero-shear-rate viscosity $\mu_{0}$ vs polymer concentration $c$ for PAA and XG

molecules are sufficiently far apart that there are minimal interactions between them and they are designated dilute in contrast with semi-dilute solutions for which $c>c^{*}$. As can be seen, $0.03 \%$ PAA corresponds almost exactly with $c=c^{*}$ whereas $0.125 \%$ PAA and $0.15 \%$ $\mathrm{XG}\left(c^{*}=0.066 \%\right)$ can both be regarded as semi-dilute.

\section{Experimental results}

\section{1. $f v s e_{W}$ and $u^{\prime} v s \operatorname{Re}_{W}$}

Following [2], we define the Fanning friction factor $f$ and Reynolds number $R e_{W}$ as follows

$f \equiv \frac{2 \tau_{W}}{\rho U_{b}^{2}} \quad$ and $\quad R e_{W} \equiv \frac{\rho U_{b} D}{\mu_{W}}$

where $\tau_{W}$ is the wall shear stress, $\rho$ is the fluid density (taken here as the same as that of the solvent, $1000 \mathrm{~kg} / \mathrm{m}^{3}$ ), $U_{b}$ is the bulk velocity determined from the mass flowrate $\dot{m}, D$ is the pipe internal diameter and $\mu_{W}$ the viscosity determined from the CarreauYasuda equation at a shear rate $\dot{\gamma}_{W}$ corresponding to $\tau_{W}$. We note that azimuthal variation in the velocity profile at any axial location must lead to azimuthal variation of the velocity gradient at the wall and hence of the wall shear stress. The values of $\tau_{W}$ estimated from the measured pressure gradient must therefore be regarded as azimuthal and streamwise average values.

We also define [9] Ekman and Rossby numbers, Ek and Ro, respectively, as

$E k \equiv \frac{\mu_{W}}{2 \Omega \rho D^{2} \sin \beta} \quad R o \equiv \frac{U_{b}}{2 \Omega D \sin \beta}$

where $\Omega$ is the angular velocity of the Earth $\left(7.3 \times 10^{-5} \mathrm{rad} / \mathrm{s}\right)$ and $\beta$ is the angle between the pipe axis and the rotation axis of the Earth ( $\beta=52^{\circ}$ for our pipe). 

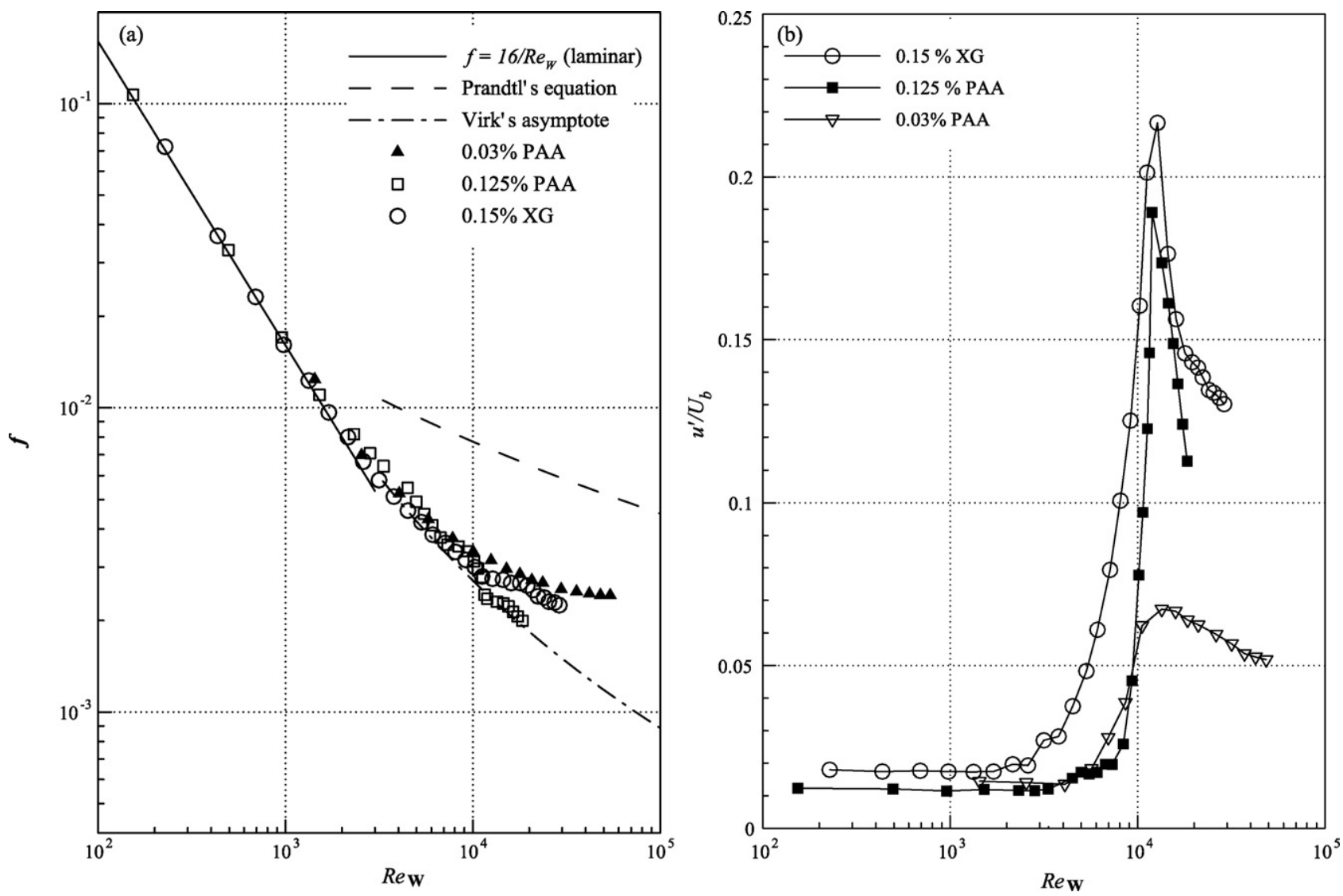

Fig. 4. (a) Fanning friction factor $f$ vs Reynolds number $R e_{W}$. (b) Near-wall fluctuation intensity $u^{\prime} / U_{b}$ vs $R e_{W}$.

For fully developed pipe flow of Newtonian fluids it is generally found that a plot of $f$ vs $R e_{W}$ shows a well-defined steep increase in $f$ from a value of about 0.007 at $R e_{W}=2300$ to a value of about 0.01 at $R e_{W}=4000$ and for practical purposes the region of increase is taken as defining the start and end of the transition from laminar to turbulent flow. As we have discussed in previous papers (e.g. [1,2]), for the flow of strongly drag-reducing polymer solutions, the $f-R e_{W}$ curve increases monotonically and no such jump occurs. However, this does not indicate that transition does not occur: if the level of rms axial-velocity fluctuations $u^{\prime}$ close to the pipe wall (typically at $80 \%$ of the pipe radius $R$ ) is monitored, an increase above the background noise level is observed indicating the start of transition. The fluctuation level then increases steeply to a maximum which is taken to indicate the end of transition. Fig. 4(a) shows the $f-R e_{W}$ data for the three non-Newtonian liquids under consideration here with the corresponding $u^{\prime} / U_{b}-R e_{W}$ data in Fig. 4(b). The delayed onset of turbulent flow for all three liquids to $R e_{W}>10^{4}$ is quite clear.

\subsection{Mean velocity distributions}

In Fig. 5 we show three velocity profiles for the 60\% glycerol solution at Reynolds numbers of 2110, 2400 and 3280 which correspond in our flow loop to the laminar, just transitional and transitional flow regimes. These profiles were measured along a horizontal diameter $\left(\theta=90^{\circ}\right)$. In each case we also include a smooth curve representing the average of the measured velocity at each radial location. That all three profiles are symmetrical is confirmed by the values of the asymmetry parameter $\alpha$, which are $0.04 \%, 0.03 \%$ and $0.04 \%$ and so well below the $2 \%$ threshold for asymmetry to be regarded as significant. These results are consistent with previous observations in our laboratory $([2,6])$ and elsewhere (e.g. [8]) that for transitional pipe flow of Newtonian fluids the mean velocity profile is invariably axisymmetric.

In Fig. 6 we show velocity profiles measured in the laminar flow regime for each of the three polymer solutions. In each case we

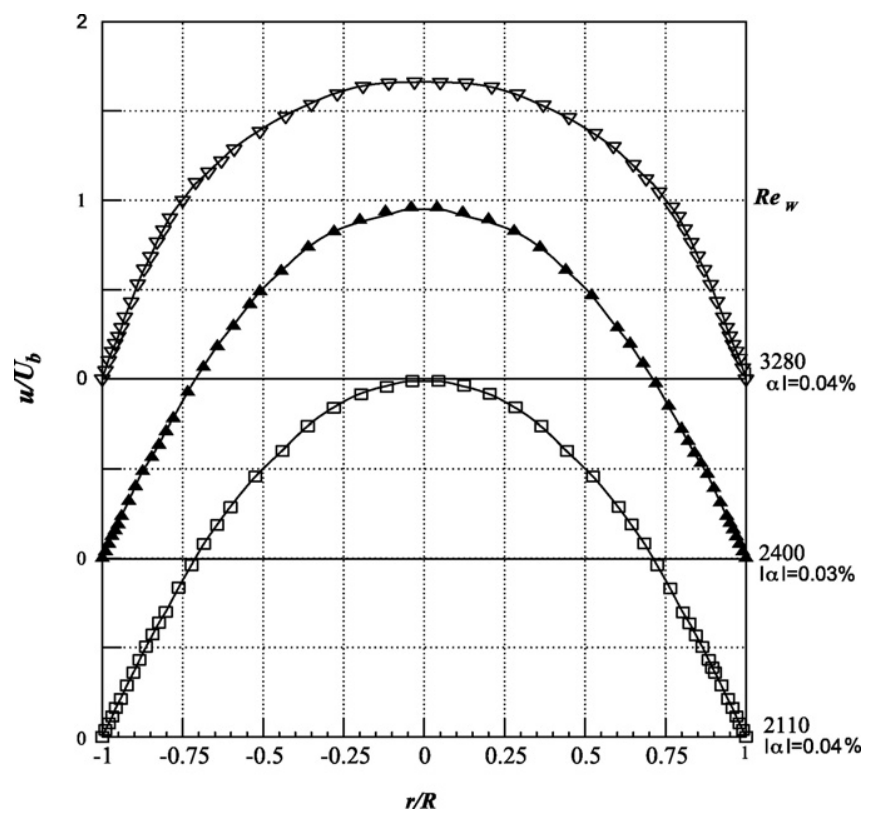

Fig. 5. Measured velocity distributions, $u / U_{b}$ vs $r / R$, along a horizontal diamete $\left(\theta=90^{\circ}\right)$ at $x / D=220$ for fully developed laminar and transitional flow of a Newtonian fluid (60\% glycerol). Solid curves represent arithmetic mean of values at the same value of $r: \operatorname{Re}_{W}=2110, \alpha=0.04 \% ; \operatorname{Re}_{W}=2400, \alpha=0.03 \% ; \operatorname{Re}_{W}=3280, \alpha=0.04 \%$. 


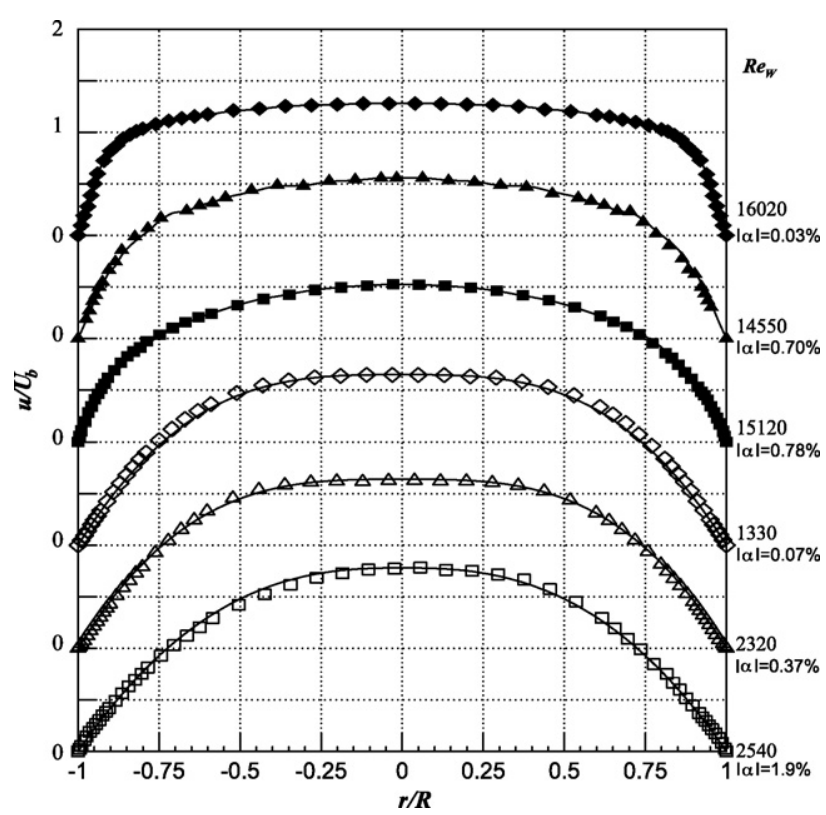

Fig. 6. Measured velocity distributions, $u / U_{b}$ vs $r / R$, along a horizontal diameter $\left(\theta=90^{\circ}\right)$ at $x / D=220$ for fully developed laminar (open symbols) and turbulent (closed symbols) flow of polymer solutions. For laminar flow solid curves represent the power-law profile and for turbulent flow the arithmetic mean of values at the same value of $r$. (a) $0.03 \% \mathrm{PAA}, \operatorname{Re}_{W}=2540, \alpha=1.9 \%, n=0.64$, (b) $0.125 \% \mathrm{PAA}$, $\operatorname{Re}_{W}=2320, \alpha=0.37 \%, n=0.47$, (c) $0.15 \% \mathrm{XG}, \operatorname{Re}_{W}=1330, \alpha=0.07 \%, n=0.48$, (d) $0.03 \%$ PAA, $R e_{W}=15,120, \alpha=0.78 \%$, (e) $0.125 \%$ PAA, $R e_{W}=14,550, \alpha=0.70 \%$, (f) $0.15 \%$ XG, $\operatorname{Re}_{W}=16,020, \alpha=0.03 \%$.

include a theoretical power-law profile (see e.g. [20]) for comparison with the power-law index $n$ selected to give the best match to the measured data: 0.03\% PAA, $R e_{W}=2540, E k=6.3, n=0.64$; 0.125\% PAA, $R e_{W}=2320, E k=23, n=0.47 ; 0.15 \%$ XG, $R e_{W}=1330$, $E k=23, n=0.48$. Only in the case of $0.03 \%$ PAA is a degree of asymmetry visually apparent and we note that this case has the lowest value of $E k=6.3$, a value low enough that the effects of the Earth's rotation become significant. The values of the asymmetry parameter $\alpha$ for the three cases are $1.9 \%, 0.37 \%$ and $0.07 \%$, respectively. Also included in Fig. 6 are three profiles for turbulent flow: $0.03 \% \mathrm{PAA}, R e_{W}=15,120, E k=3.2, R o=4900, \alpha=0.78 \%$; 0.125\% PAA, $R e_{W}=14,550, E k=9.5, R o=1.5 \times 10^{5}, \alpha=0.70 \% ; 0.15 \%$ $\mathrm{XG}, R e_{W}=16,020, E k=6.9, R o=1.2 \times 10^{5}, \alpha=0.03 \%$. As anticipated, in no case is there perceptible asymmetry in the turbulent flow profiles.

Fig. 7 shows a series of transitional-regime $\left(R e_{W}=5780\right)$ velocity profiles for the flow of $0.03 \%$ PAA measured at $x / D=220$ along four different diameters: $\theta=0^{\circ}, 45^{\circ}, 90^{\circ}$ and $135^{\circ}$, all angles measured from the vertical (see Fig. 1 ). As can be seen, the degree of asymmetry varies quite significantly with $\theta$ and is greatest $\left(\alpha=7 \%\right.$ ) for $45^{\circ}$. For $0.125 \%$ PAA at $R e_{W}=10,140$, also within the transition regime, the picture (Fig. 8) is even clearer with $\alpha$ varying between $1.8 \%$ at $\theta=120^{\circ}$ and $18 \%$ at $\theta=45^{\circ}$. As remarked in Section 2, each profile took approximately 3 to $4 \mathrm{~h}$ to measure so the results represent long-time averages and there is no possibility that the asymmetry was precessing rather than being spatially "frozen". The variation in $\alpha$ with $\theta$ for the two sets of profiles is seen in Fig. 9. Although the maxima/minima occur at the same $\theta$ values $\left(45^{\circ}\right.$ and $\left.225^{\circ}\right)$ there is roughly $33^{\circ}$ between the zero crossings. In recent work [12] on flow of a yield-stress fluid, the zero crossings were found to line up quite precisely and the magnitude of the asymmetry to evolve smoothly with axial distance. As in the present study, it was found that the peak asymmetry always had the same angular location and as a result the conclusion drawn in that study was that

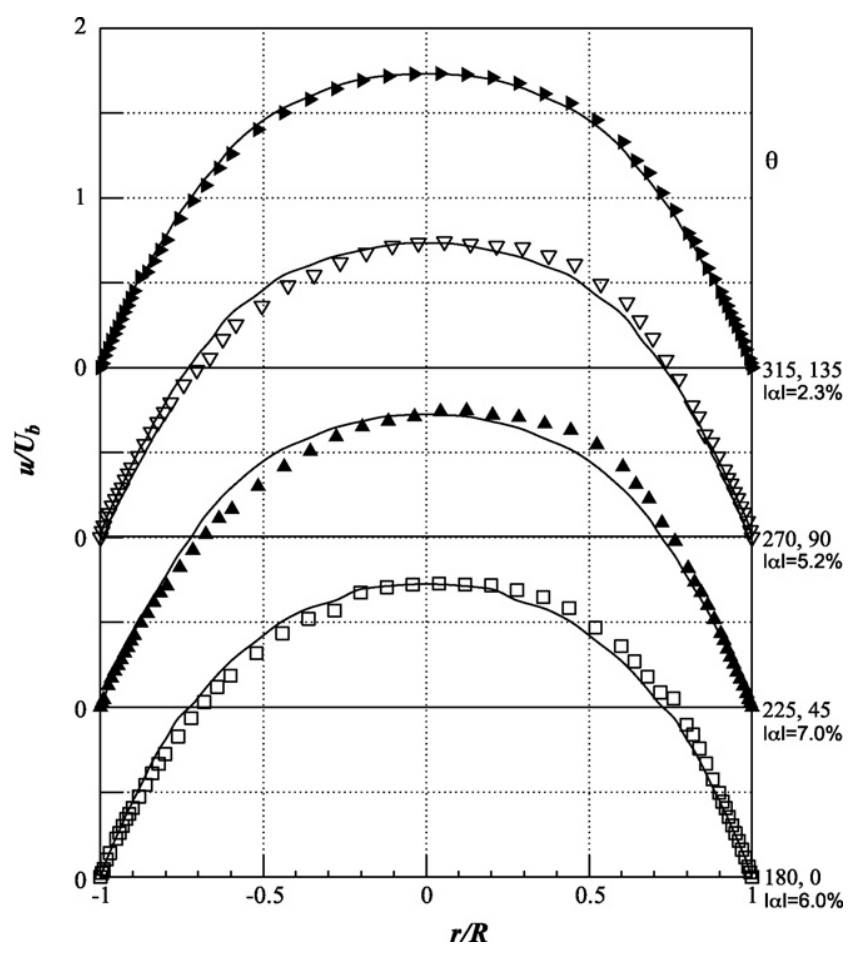

Fig. 7. Measured velocity distributions for $\theta=0^{\circ} \quad(\alpha=6.0 \%), 45^{\circ} \quad(\alpha=7.0 \%), 90^{\circ}$ $(\alpha=5.2 \%)$ and $135^{\circ}(\alpha=2.3 \%)$ at $x / D=220$ for transitional flow of $0.03 \%$ PAA at $R e_{W}=5780$

the sense of the asymmetry depends probably only on the inlet conditions.

Transitional-flow profiles for $0.15 \%$ XG at $R_{W}=10,260$ (Fig. 10) show a very similar trend to those for PAA, once again with the maximum $(\alpha=15 \%)$ at $45^{\circ}$. The highest asymmetry reported in [12] for the transitional flow of Carbopol is considerably less than observed

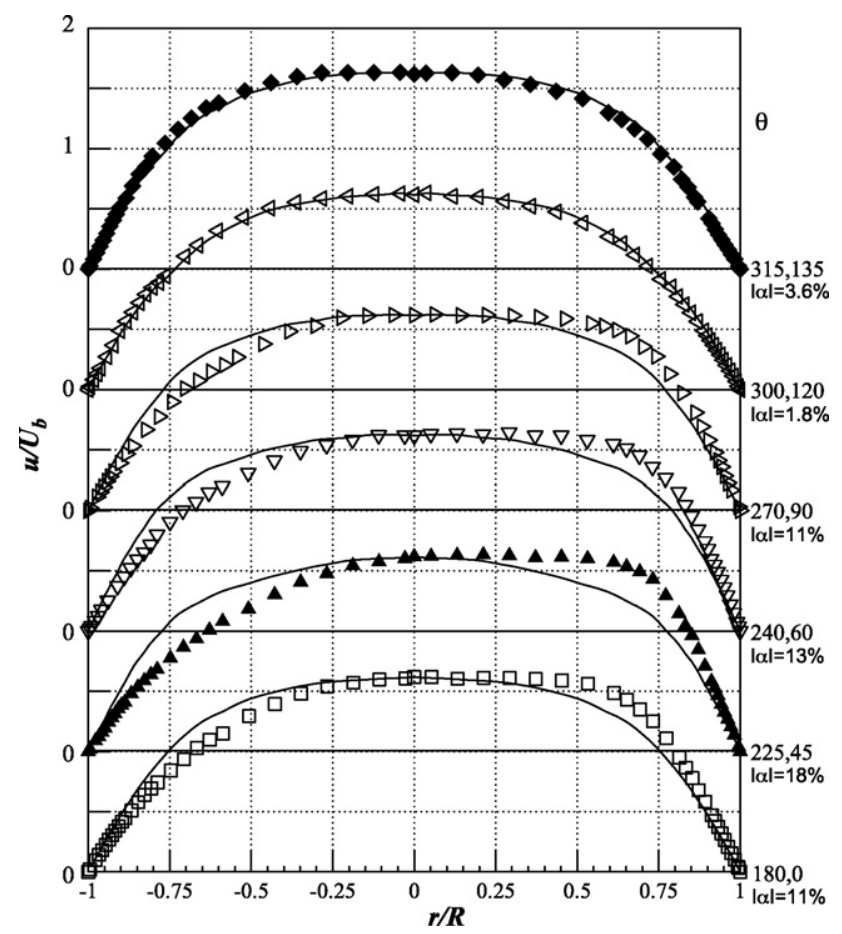

Fig. 8. Measured velocity distributions for $\theta=0^{\circ} \quad(\alpha=11 \%), 45^{\circ} \quad(\alpha=18 \%), 60$ $(\alpha=13 \%), 90^{\circ}(\alpha=11 \%), 120^{\circ}(\alpha=1.8 \%)$ and $135^{\circ}(\alpha=3.6 \%)$ at $x / D=220$ for transitional flow of $0.125 \%$ PAA at $R e_{W}=10,140$ 


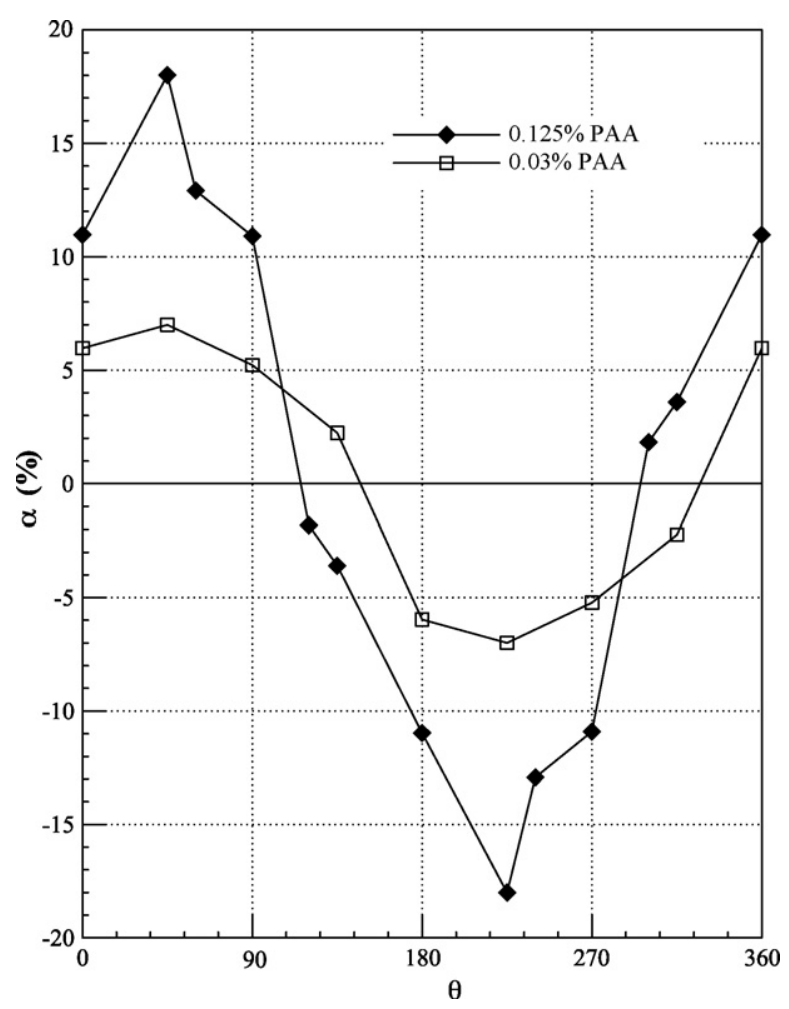

Fig. 9. Azimuthal variation of asymmetry parameter $\alpha(\theta)$ at $x / D=220$ for transitional flow of $0.03 \%$ PAA $\left(R e_{W}=5780\right)$ and $0.125 \%$ PAA $\left(R e_{W}=10,140\right)$.

here. In terms of the streak velocity at $r / R=0.72$, we see values as high as $0.4(0.15 \% \mathrm{XG})$ and $0.5(0.125 \%$ PAA) whereas the highest values in [12] are about 0.2 . The difference may be due to the much higher Reynolds numbers here.

The observations in Figs. 7-10 raise a number of questions. The first is whether the asymmetry stems from an upstream dis-

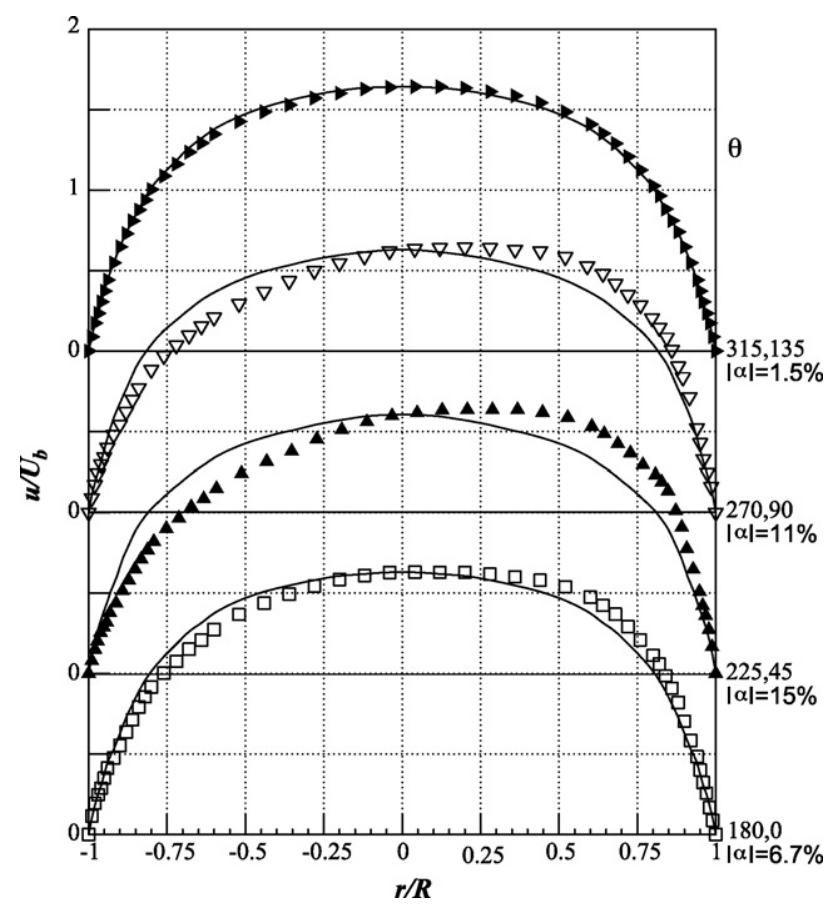

Fig. 10. Measured velocity distributions for $\theta=0^{\circ} \quad(\alpha=6.7 \%), 45^{\circ} \quad(\alpha=15 \%), 90^{\circ}$ $(\alpha=11 \%)$ and $135^{\circ}(\alpha=1.5 \%)$ at $x / D=220$ for transitional flow of $0.15 \%$ XG at $\operatorname{Re}_{W}=10,260$. turbance or develops with axial location. If there is an upstream influence, the observations for the flow of $60 \%$ glycerol in our flow facility (Fig. 5) show that it does not lead to asymmetry for the transitional flow of a Newtonian liquid. Figs. 11 and 12 show major differences between the three flows of XG and PAA at the first measuring location, $x / D=15$, just downstream of the plenum chamber. In fact, the differences are quite remarkable. The first profile for $0.15 \%$ XG (Fig. 11(c)) is practically flat in the pipe centre $(r / R<0.5)$, while the two PAA flows show a significant momentum deficit, particularly $0.125 \%$ PAA where the peak velocity is $30 \%$ higher than the centreline velocity (Fig. 11(b)). All three flow start out with little asymmetry but the asymmetry development with downstream distance is very clear. This development is quantified in terms of $\alpha(\theta$, $x / D$ ) in Fig. 12. The data in Fig. 12(a) for 0.03\% PAA is quite different in character from that for 0.125\% PAA in Fig. 12(b) and 0.15\% XG in Fig. 12(c). The low concentration PAA flow starts out at $x / D=15$ with a very low level of asymmetry with a maximum and a minimum at $\theta=90^{\circ}$ and $270^{\circ}$, respectively. By $x / D=65$ the asymmetry is well-defined (maximum $\alpha=3 \%$ ) but the minimum is now at $\theta=90^{\circ}$ and the maximum at $270^{\circ}$. However, by $x / D=160$ the asymmetry appears to have reached its final state with a maximum $\alpha=7 \%$ at $45^{\circ}$. Thus both the location and the magnitude of the peak asymmetry evolve with axial location. These data for $0.03 \%$ PAA are particularly significant because they show that the asymmetry can evolve both azimuthally as well as axially. The higher concentration of PAA exhibits negligible levels of asymmetry until $x / D=200$ by which location the $\alpha(\theta)$ variation is very similar in shape to those for $0.03 \%$ PAA but with considerably higher maxima ( $\alpha=18 \%$ ). For $0.15 \%$ XG the asymmetry develops slightly earlier than for the $0.125 \%$ PAA. There is nothing in the observations of any of the fluids investigated to suggest the asymmetry is initiated by a disturbance far upstream, nevertheless we explore further the upstream influence below.

\section{Influence of changes to upstream and downstream conditions}

\subsection{Upstream disturbance}

As has been seen, the highest level of asymmetry observed was for velocity profiles measured along a radial line at $45^{\circ}$ to the vertical, with the highest flow velocities in the top right hand quadrant of the pipe (see Fig. 1). The holes in the plenum chamber (see Fig. 1) above a diametral line between $135^{\circ}$ and $315^{\circ}$ were blanked-off to explore the effect of creating higher velocities at inlet to the pipe in the lower left hand quadrant. As would be expected, the first effect of this imposed disturbance was to move the transition region to lower Reynolds numbers: the peak values of near-wall fluctuation intensity occur at about $R e_{W}=8000$ for the blanked-off disc compared with about 13,000 for the disc with uncovered holes. At $x / D=220$, a flow of $0.15 \%$ XG at $R e_{W}=10,260$ for the blanked-off disc was found to be slightly asymmetric (maximum $\alpha=3.2 \%$ ) in the same sense as for the open disc. In other words, the initial asymmetry caused by the blanked-off disc had been reversed. It is probable that at $x / D=220$ the flow was still recovering from the upstream disturbance and the "final" asymmetry would have been higher if the pipe run was further extended.

A set of profiles, again for $0.15 \% \mathrm{XG}$, at $R e_{W}=7120$, in the transition regime (Fig. 13), shows the reversal and continued development of asymmetry from $x / D=160(\alpha=9.2 \%)$ to $x / D=220$ $(\alpha=12 \%)$. These profiles were measured along a horizontal diameter $\left(\theta=90^{\circ}\right)$. Although not shown here, the asymmetry was even higher (maximum $\alpha=15 \%$ ) at $\theta=45^{\circ}$. We limited this series of experiments to XG as it is less prone to mechanical degradation than PAA and the blanked-off baffle would lead to higher levels of shearing than 

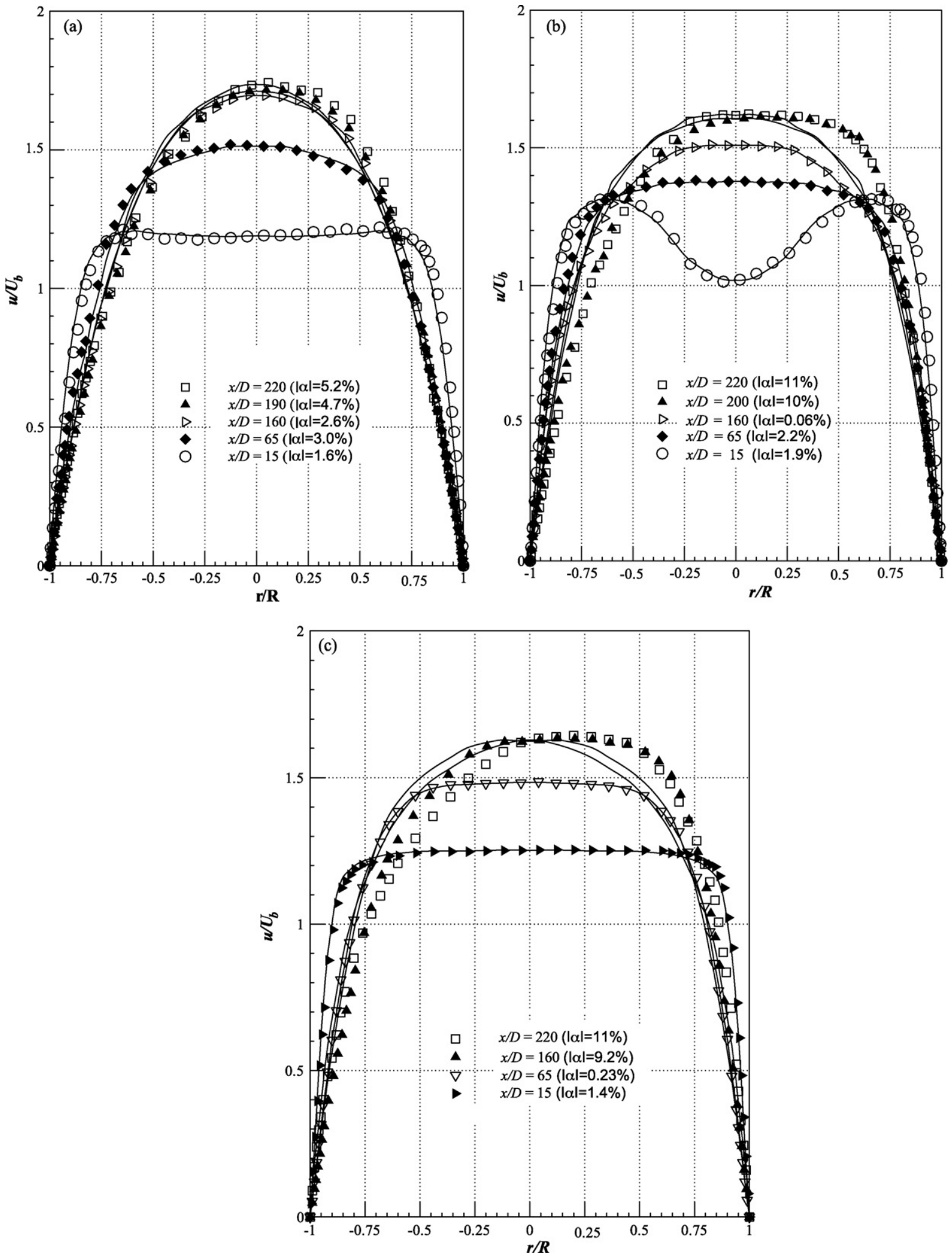

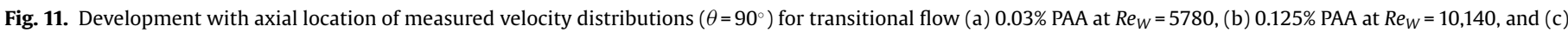
$0.15 \%$ XG at $R e_{W}=10,260$. 

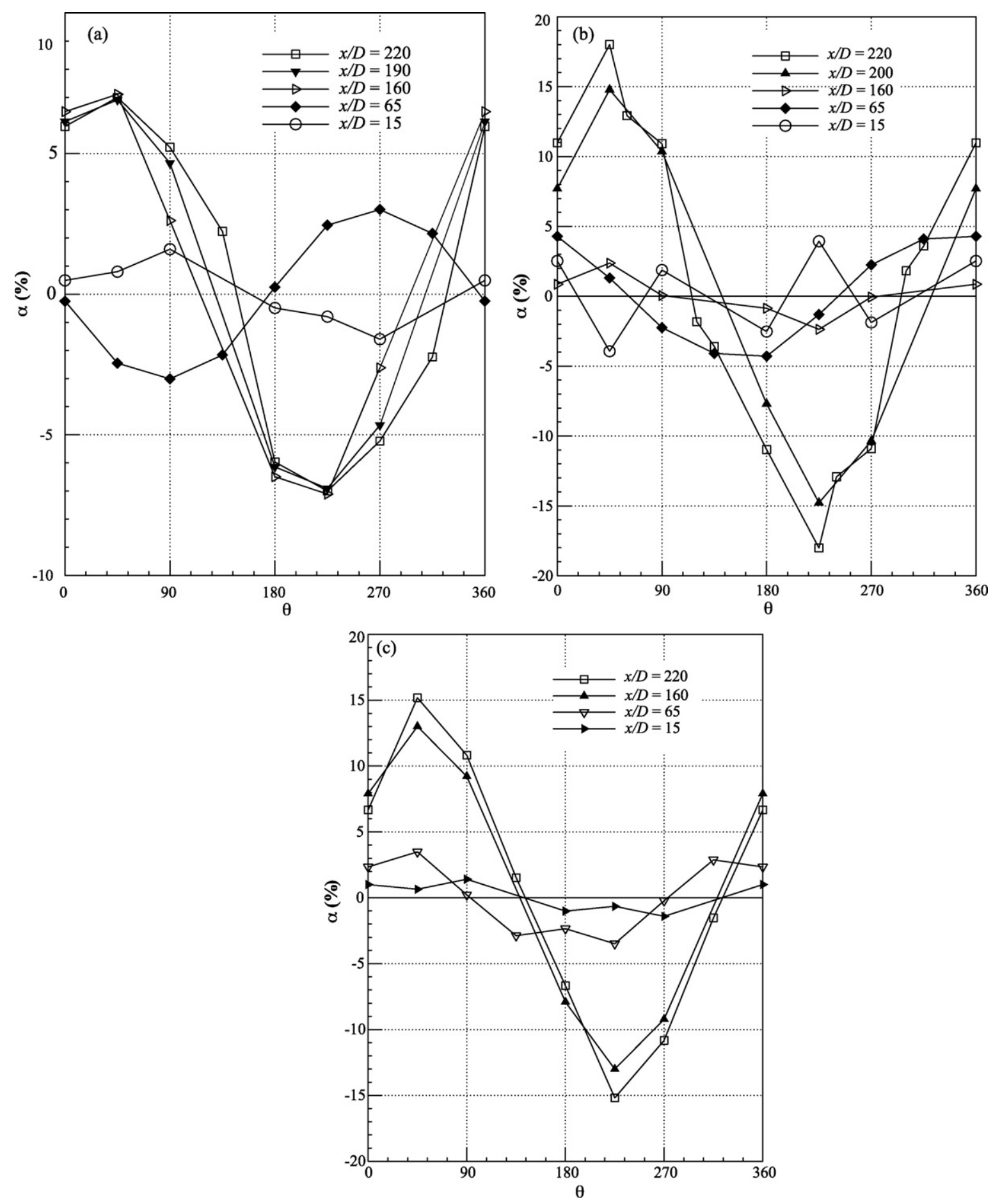

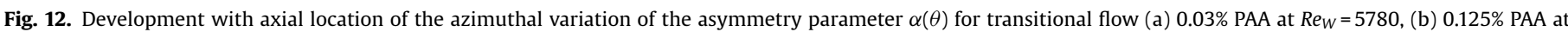
$\operatorname{Re}_{W}=10,140$, and (c) $0.15 \%$ XG at $\operatorname{Re}_{W}=10,260$.

the standard geometry. There seems to be no reason to believe that the behaviour of XG is atypical and we suggest these observations reinforce our view that the asymmetry in transitional flow cannot be a consequence of minor geometric imperfections far upstream.

\subsection{Downstream disturbance}

There is a sharp (radius $\sim 200 \mathrm{~mm}$, i.e. $\sim 2 D$ ) $90^{\circ}$ bend angled at approximately $255^{\circ}$ to the vertical at the downstream end of the pipe run $(x / D=232), 12$ diameters downstream of the final measuring location. To see whether a disturbance could be propagating upstream from the bend causing asymmetry in the flow, the bend was turned anticlockwise through $90^{\circ}$ to the $165^{\circ}$ position. Within experimental uncertainty, this change was found to have no affect on the flow asymmetry.

\section{Axial-velocity fluctuations}

Profiles of the radial variation of the rms axial-velocity fluctuations $u^{\prime}(r)$, corresponding to the mean velocity profiles (horizontal diameter, $\theta=90^{\circ}$ ) in Figs. 7-9 are shown in Figs. 14-16. The profiles are shown normalised using both the bulk velocity $U_{b}$ (Figs. 


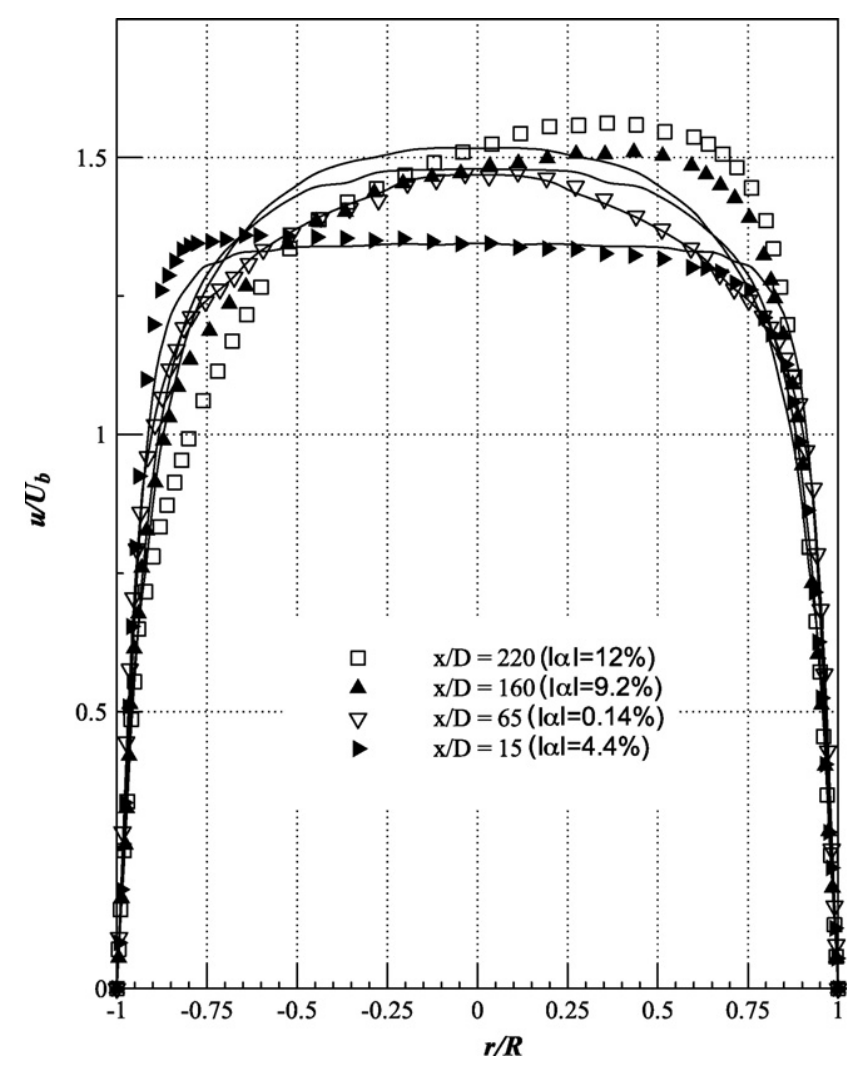

Fig. 13. Development with axial location of measured velocity distributions $\left(\theta=90^{\circ}\right)$ for transitional flow of $0.15 \%$ XG at $R e_{W}=7120$ with plenum disc partially blocked.

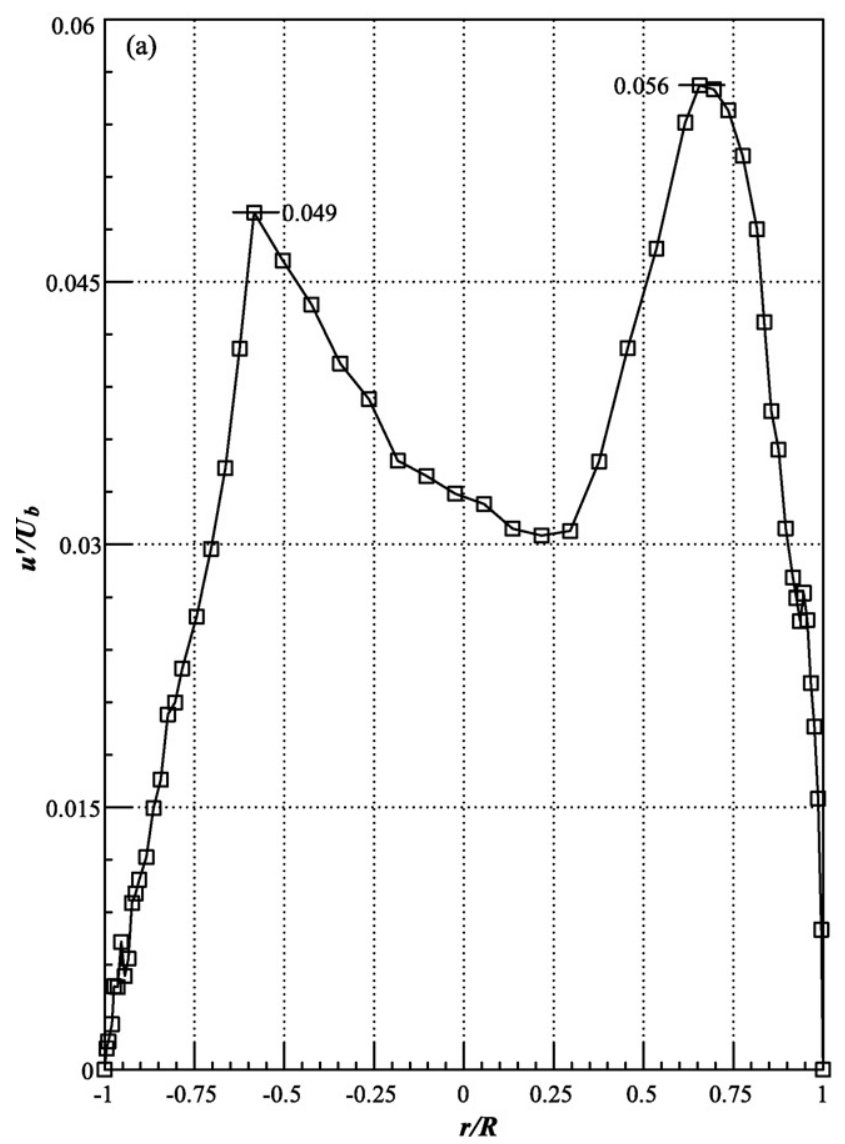

14(a), 15(a) and 16(a)) and the local mean velocity $u$ (Figs. 14(b), 15(b) and 16(b)). It is immediately apparent that the absolute level of $u^{\prime}$ (i.e. normalised with $U_{b}$ which is constant for any given flow) is skewed in the same way as the mean velocity $u$. However when $u^{\prime}$ is normalised by the local mean velocity $u$ the picture looks quite different. For 0.03\% PAA, Fig. 14(b), there is a well-defined peak in $u^{\prime} / u$ on the low-velocity side at $r / R=-0.65$ at the same radial location as for $u^{\prime} / U_{b}$ whereas on the high-velocity side the peak at $r / R=0.65$ has disappeared and the fluctuations increase essentially monotonically to a peak in the immediate vicinity of the pipe wall $(r / R=0.95)$. For $0.125 \%$ PAA, the peak locations observed in Fig. 15(a) are preserved in Fig. 15(b) but the $u^{\prime} / u$ peak is higher on the low-velocity side whereas the $u^{\prime} / U_{b}$ peak is higher on the high-velocity side. The picture for $0.15 \%$ XG is different again with the $u^{\prime} / u$ distribution showing no peak on either side of the axis whereas the $u^{\prime} / U_{b}$ distribution shows two very well-defined peaks. The conclusion seems to be that, as would be expected, the higher fluctuation levels coincide with the regions of higher velocity gradient (i.e. shear) rather than high local velocity.

The experimental observations under discussion here reveal a potential problem with the technique we have long advocated [1] for detection of the onset of transition. As can be seen from Fig. 14(a), monitoring the near-wall velocity fluctuations on one side of the pipe would lead to quite different conclusions than doing so on the other side: at $r / R=-0.8, u^{\prime} / U_{b} \approx 0.02$ which is at the level of the background noise and would be interpreted as indicating the flow is still laminar. At $r / R=0.8$, however, $u^{\prime} / U_{b} \approx 0.05$ and the flow is clearly transitional. We conclude that ideally, the near-wall fluctuations should be monitored at at least two locations $90^{\circ}$ apart. It is fortunate that in all of our experiments the measurement location was on the side of the pipe where the fluctuation intensities were relatively high.

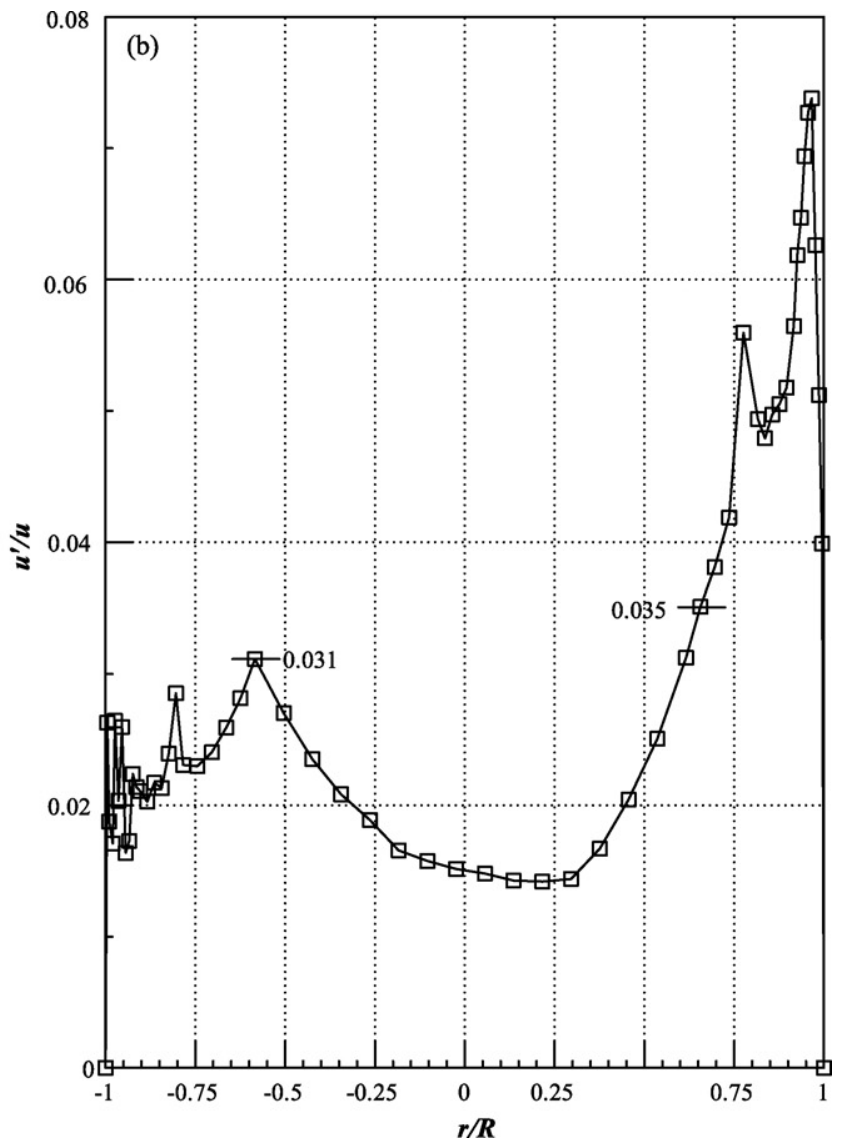

Fig. 14. RMS axial-velocity fluctuations $u^{\prime}$ at $x / D=220$ and $\theta=90^{\circ}$ for transitional flow of $0.03 \%$ PAA at $R e_{W}=5780$ (a) $u^{\prime}$ normalised with $U_{b}$ and (b) $u^{\prime}$ normalised with $u$. 

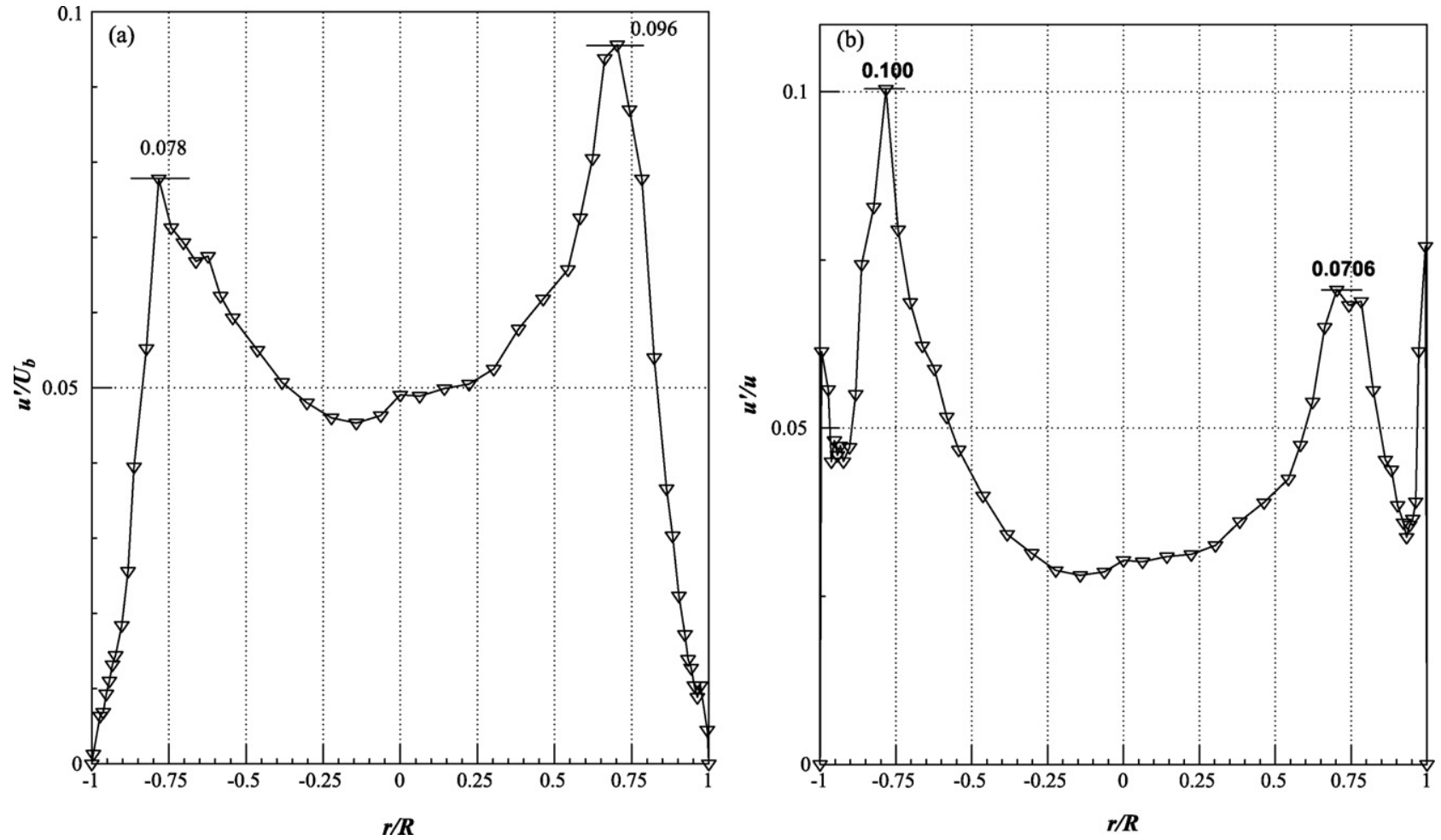

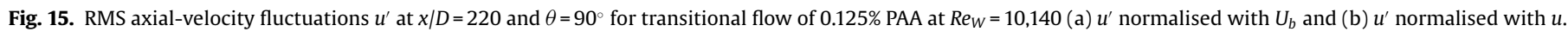
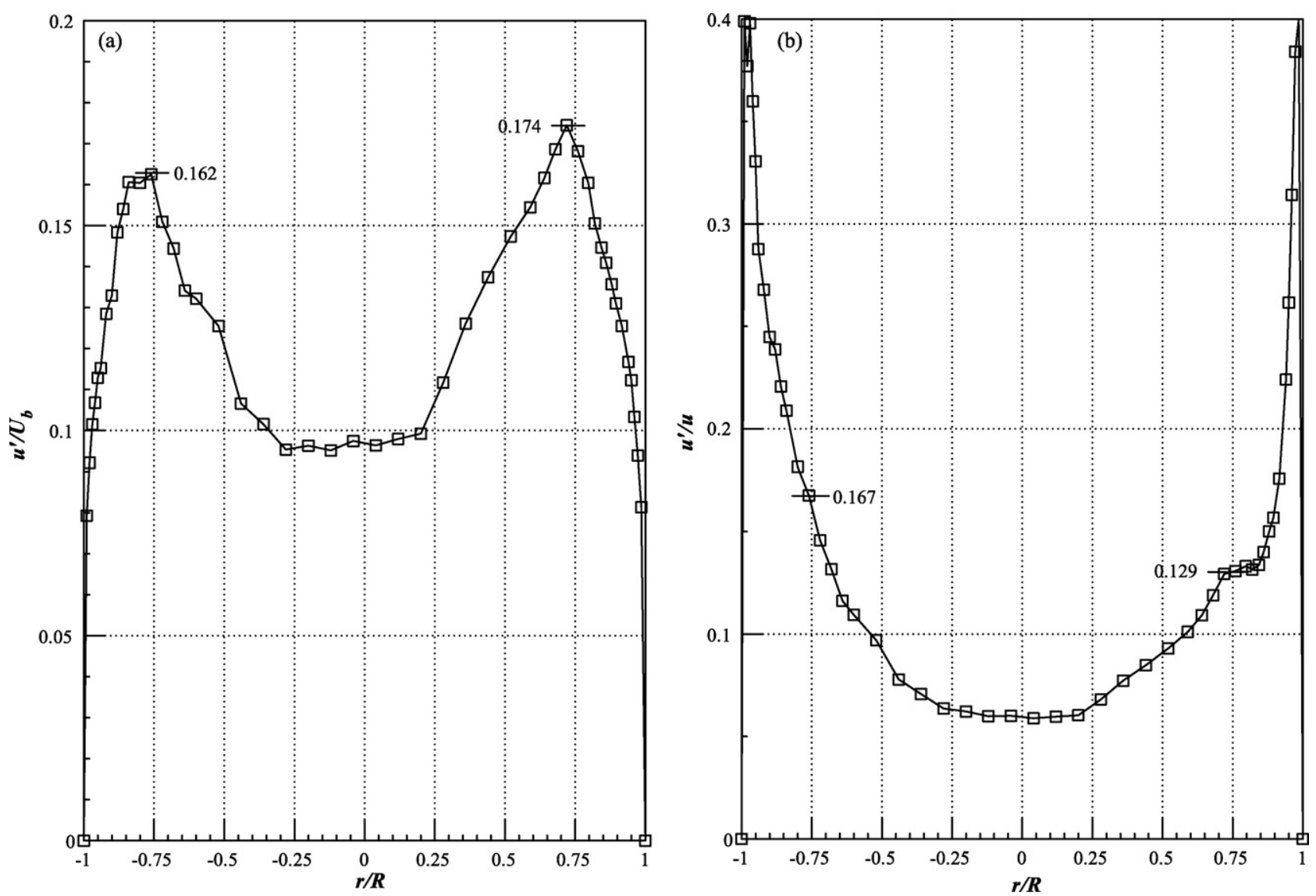

Fig. 16. RMS axial-velocity fluctuations $u^{\prime}$ at $x / D=220$ and $\theta=90^{\circ}$ for transitional flow of $0.15 \%$ XG at $R e_{W}=10,260$ (a) $u^{\prime}$ normalised with $U_{b}$ and (b) $u^{\prime}$ normalised with $u$. 


\section{Conclusions}

Asymmetry in transitional pipe flow of viscoelastic, shearthinning polymers, including those exhibiting a yield stress has been well documented by several investigators $[2,3,7,8,12]$ since it was first observed just over a decade ago [1] and the existence of this phenomenon is now incontrovertible. Although the present study is the most systematic and extensive to date, it has not led to an explanation of the physical mechanism which triggers the asymmetry. In other recent work [12] it was tentatively concluded that the sense of the asymmetry depended on the initial conditions. In contrast, our experiments appear to rule out a number of possible explanations, including the direct influence of flow distortion due to the flow geometry far upstream or downstream. Both rotation of the Earth and curvature of the pipe axis can also be ruled out: the Rossby numbers are too high for the former and the Dean numbers (no higher than 10, here) too low for the latter. As others have commented [12], the asymmetry suggests the existence of a coherent structure characterised by two counter-rotating longitudinal vortices, but the origin of these vortices remains unexplained. We observe that velocity fluctuations are highest in regions of high shear, as is to be expected, rather than in regions of high velocity but the distributions of fluctuation intensity are similar to those of the mean velocity.

\section{References}

[1] M.P. Escudier, F. Presti, Pipe flow of a thixotropic liquid, J. Non-Newtonian Fluid Mech. 62 (1996) 291-306.

[2] M.P. Escudier, RJ. Poole, F. Presti, C. Dales, C. Nouar, C. Desaubry, L. Graham, L. Pullum, Observations of asymmetrical flow behaviour in transitional pipe flow of yield-stress and other shear-thinning liquids, J. Non-Newtonian Fluid Mech. 127 (2005) 143-155.
[3] J. Peixinho, C. Nouar, C. Desaubry, B. Théron, Laminar transitional and turbulent flow of yield stress fluid in a pipe, J. Non-Newtonian Fluid Mech. 128 (2005) $172-184$.

[4] S. Eliahou, A. Tumin, I. Wygnanski, Laminar-turbulent transition in Poiseuille pipe flow subjected to periodic perturbation emanating from a wall, J. Fluid Mech. 361 (1998) 333-349.

[5] J.M.J. den Toonder, F.T.M. Nieuwstadt, Reynolds number effects in a turbulent pipe flow for low to moderate Re, Phys. Fluids 9 (11) (1997) 3398-3409.

[6] F. Presti, Investigation of transitional and turbulent pipe flow of non-Newtonian fluids, Ph.D. thesis, University of Liverpool, 2000.

[7] B. Güzel, I. Frigaard, D.M. Martinez, Predicting laminar-turbulent transition in Poiseuille pipe flow for non-Newtonian fluids, Chem. Eng. Sci. 64 (2009) 254-264.

[8] B. Güzel, T.Burghelea, I. Frigaard, D.M. Martinez, Observations of laminarturbulent transition of a yield stress fluid in Hagen-Poiseuille flow, J. Fluid Mech., in press.

[9] A.A. Draad, F.T.M. Nieuwstadt, The Earth's rotation and laminar pipe flow, J. Fluid Mech. 361 (1998) 297-308.

[10] W.R. Dean, Note on the motion of a fluid in a curved pipe, Philos. Mag. 4 (1927) 208.

[11] F.T. Pinho, J.H. Whitelaw, Flow of non-Newtonian fluids in a pipe, J. NonNewtonian Fluid Mech. 34 (1990) 129-144.

[12] A. Esmael, C. Nouar, Transitional flow of a yield-stress fluid in a pipe: evidence of a robust coherent structure, Phys. Rev. (2008), E 77057302.

[13] P. Dimotakis, Single scattering particle laser-Doppler measurements of turbulence. Applications of non-intrusive instrumentation in fluid flow research, AGARD Conf. N.193, paper 10, 1976.

[14] K. Walters, A.Q. Bhatti, N. Mori, The influence of polymer conformation on the rheological properties of aqueous polymer solutions, in: D. de Kee, P.N. Kaloni (Eds.), Recent Developments in Structured Continua, Pitman, 1990.

[15] M.P. Escudier, F. Presti, S. Smith, Drag reduction in the turbulent pipe flow of polymers, J. Non-Newtonian Fluid Mech. 81 (1999) 197-213.

[16] P.S. Virk, H.S. Mickley, K.A. Smith, The ultimate asymptote and mean flow structure in Tom's phenomenon, J. Appl. Mech. 37 (1970) 488-493.

[17] K. Yasuda, R.C. Armstrong, R.E. Cohen, Shear flow properties of concentrated solutions of linear and star branched polystyrenes, Rheol. Acta 20 (1981) 163-178.

[18] F. Keegan, Private communication, 2008.

[19] A. Jaffar-Jaaper, Private communication, 2008.

[20] R.B. Bird, R.C. Armstrong, O. Hassager, Dynamics of polymeric fluids Fluid Mechanics, vol. 1, John Wiley \& Sons, 1987. 